\title{
Progress in the Treatment of Ewing Sarcoma: Are the Rumors of the Demise of Cytotoxic
} Chemotherapy Premature?

Fortschritte bei der Behandlung von Ewing-Sarkomen: Hat die zytotoxische Chemotherapie das Ende der Fahnenstange erreicht?

G. Rosen

Bibliography

DOI http://dx.doi.org/

$10.1055 / \mathrm{s}-0035-1548751$

Klin Padiatr 2015; 227: 105-107

(c) Georg Thieme Verlag KG

Stuttgart · New York

ISSN 0300-8630

Correspondence
Gerald Rosen, MD

Professor of Medicine

NYU School of Medicine

Director of Adult Sarcoma

Program

NYU Clinical Cancer Center

160 E. $34^{\text {th }}$ St, New York, NY 10016

Tel.: + 1/212/7315758

Cell: 6464838335

Fax: + 1/212/7315646

Gerald.rosen@nyumc.org
In this issue, Bollling et. al. review the development of treatment of Ewing sarcoma as it evolved over the past 30 years of clinical trials in Europe, largely under the leadership of Heribert Jurgens to whom this review is dedicated. The 44 authors were teachers, colleagues, students and co-investigators of Jurgens. The authors attribute the ability to make progress in the treatment of Ewing sarcoma through the establishment of larger and still larger cooperative studies in order to demonstrate statistically significant advances in the treatment of this rare disease.

Thus, starting with the German/Austrian cooperative Ewing sarcoma study (CESS) and later joining other European groups to form the European Intergroup Cooperative Ewing Sarcoma Study (EICESS) and finally the "European Ewing tumor Working Initiative of National Groups" or "EURO-EWING" conducted trials in the treatment of this rare bone tumor.

By the early 1980s, 4 drugs including vincristine, dactinomycin, cyclophosphamide, and doxorubicin were almost universally accepted for chemotherapy of Ewing sarcoma $[9,13]$. However under the leadership of Heribert Jurgens important further advances made by the CESS studies included the finding that ifosfamide increased the event-free survival of high risk patients and indeed those studies defined the high risk and standard risk groups. The results of CESS 86 included 301 patients treated from 1986 through 1992 and were not published until 2001, and the overall event-free survival was $52 \%$. Fifty two percent however was a 10 year event-free survival and as such represented the standard achievable cure rate for Ewing sarcoma [11]. The better long term outcome for high risk patients was attributed to not only the addition of ifosfamide, but the better use of multimodality treatment including a surgical imperative for the primary tumor. The latter approach to the primary tumor was also of great value for the standard (or low) risk patient.

The EICESS-92 study randomized 647 patients. Of 155 standard risk patients half had ifosfamide $\left(6 \mathrm{~g} / \mathrm{m}^{2}\right)$ containing maintenance chemotherapy, and half had cyclophosphamide containing maintenance, although all had 4 courses of preoperative (or induction) ifosfamide containing chemotherapy. The study concluded that there was no difference in the 2 regimens for the standard risk patients who all achieved an event-free survival (EFS) of approximately $75 \%$. However, the majority of patients in this study (492/647) were high risk patients and they all had the ifosfamide regimen for 14 courses but half of those high risk patients received etoposide in addition. The latter results showed a significant (but not dramatic) increase in EFS in the etoposide treated group (52 vs. $47 \%$ ) [12]. The difference was in the range of the estimated long term risk of leukemia associated with etoposide treatment [6].

The substitution of cyclophosphamide for ifosfamide in standard risk patients was supposedly desirable to prevent renal damage by ifosfamide. Ifosfamide renal tubular toxicity, although present to some degree in almost $100 \%$ of patients treated with ifosfamide doses in excess of $9 \mathrm{~g} / \mathrm{m}^{2}$, is easily treated with electrolyte replacement therapy and is not always permanent or of clinical significance. Nevertheless to drive the point home with more statistical significance, the EURO-EWING99-R1 trial screened roughly 2500 Ewing sarcoma patients to randomize 856 standard risk patients to the cyclophosphamide vs. ifosfamide maintenance regimens. This time, after more than a decade later, again no difference was noted between the 2 regimens with approximately $25 \%$ of the standard risk patients in each group destined to die of Ewing sarcoma [7]. This result coupled with the death rate of the high risk patients still around $50 \%$ is where we are at today with little improvement over the outcomes of 20 years ago.

Indeed the authors of this review point out that the results for localized Ewing sarcoma have not improved significantly over the past 2 decades, and in addition, are still disappointingly poor in patients who present with disseminated disease. They further imply that we have reached the limit of what we can achieve with cytotoxic chemotherapy save for a few regimens with irinotecan or topotecan that combined with an alkylating agent have been known to produce evanescent responses in relapsed patients [2]. They imply that the future of Ewing sarcomas improved outcome may lie in the discovery of biomarkers that can help better stratify patients risk, and then presumably adding the appropriate targeted therapy to help improve that patient's outcome. Those of us familiar with targeted therapies are aware that their addition has not produced much in the way of cures, and they usually only extend survival by slowing down tumor growth. The place for targeted therapy may be best suited for patients with metastatic disease that might benefit from greater overall survival with a much better quality of life than that achievable with maintenance chemotherapy. The prospect of further risk stratification with the addition of new targeted therapy does not seem like the best or only option that can increase the cure rate of 
Ewing sarcoma in the near foreseeable future. Unless studies are fast tracked in some way, we may be decades away from improving results in rare tumors like Ewing sarcoma with the traditional investigative approach. Discovery (a giant step forward) rather than protracted investigation (producing a quantum advance) is needed in the design of new clinical trials.

I don't think we are done with cytotoxic therapy. There is still much more to be gained from this modality. Ifosfamide for instance may have much greater potential value than has been demonstrated in the EURO-Ewing 99 study. Many of us have used ifosfamide in doses between 9 and $14 \mathrm{~g} / \mathrm{m}^{2}$ (and higher) alone or in combination with doxorubicin, to treat the more resistant adult soft tissue spindle cell sarcomas, and to treat metastatic osteosarcoma in children and adolescents. While all such patients may experience renal tubular acidosis needing bicarbonate and potassium replacement, very few have permanent or clinically significant renal impairment. Indeed Craft reported increasing the EFS of Ewing sarcoma patients from 44 to $62 \%$ by increasing the dose of ifosfamide from $6 \mathrm{~g} / \mathrm{m}^{2}$ to $9 \mathrm{~g} / \mathrm{m}^{2}$ in the induction phase of treatment. Of the 243 patients treated in that single arm trial presumably 45 patients' life were saved by the $50 \%$ increase in the dose of ifosfamide [3]. Patel (M.D. Anderson Hospital) also noted a dose response relationship for in treating metastatic bone and soft tissue sarcomas up to the dose of $14 \mathrm{~g} /$ $\mathrm{m}^{2}$ [10]. In my experience, I have treated bone (Ewing and osteogenic sarcoma) as well as soft tissue sarcomas with ifosfamide at the dose of $14-18 \mathrm{~g} / \mathrm{m}^{2}$ for since 1990 [4]. The majority with primary tumor and were long term survivors. Although all required electrolyte replacement (which is built into the treatment protocol) permanent renal impairment was limited to adults over 50 years of age that started with pretreatment creatinine elevations. After therapy the renal tubular function in those few patients improved enough so that no one required ongoing permanent treatment for renal tubular acidosis. Of course an elevated creatinine during treatment requires individual dosage modification. Usually the dose is kept the same but administered over a protracted period. Other times we have stopped the ifosfamide at the dose of $10 \mathrm{~g} / \mathrm{m}^{2}$ and added doxorubicin (60-75 $\left.\mathrm{mg} / \mathrm{m}^{2}\right)$. In treating at the dose of $18 \mathrm{~g} / \mathrm{m}^{2}$ we observe dramatic responses in Ewing sarcoma, but we never give more than 4 doses for a cumulative dose of only $72 \mathrm{~g} / \mathrm{m}^{2}$. There are reports of long term renal toxicity in children receiving large doses of ifosfamide $[14,15]$. However that toxicity occurred with cumulative doses in excess of $84 \mathrm{~g} / \mathrm{m}^{2}$.

Higher doses of ifosfamide may be safely given with no clinically significant long term toxicity, if not excessively repeated to large cumulative doses in excess of $84 \mathrm{~g} / \mathrm{m}^{2}$. Considering a potential increase in the EFS of almost $20 \%$ with a modest increase in ifosfamide dose [3], further escalation and intensification of ifosfamide therapy while reducing the total cumulative dose might be the best way to proceed in the immediate future. Currently in the EURO-EWING99 trial, high risk patients receive a cumulative ifosfamide dose of $102 \mathrm{~g} / \mathrm{m}^{2}$, the majority of which is given in the protracted consolidation (maintenance) phase at the less effective dose of $6 \mathrm{~g} / \mathrm{m}^{2}$. Would more intensive higher dose ifosfamide, but a lower cumulative dose result in a higher EFS with less renal toxicity? Indeed Granowetter reported a Children's Cancer Study Group study comparing Intensive alkylating agent against lower dose alkylating agent therapy in Ewing sarcoma [5]. The ifosfamide dose of $9 \mathrm{~g} / \mathrm{m}^{2}$ was compared to $12 \mathrm{~g} / \mathrm{m}^{2}$ in the intensive therapy arm. The study showed no difference in EFS for each group, however the overall EFS for all of the patients was $75 \%$. This compares favorably to the EICESS-92 study ${ }^{4}$ where the overall EFS for all patients (standard and high risk) was in the range of only $58 \%$. Perhaps this line of enquiry can be tweaked to produce an even better EFS.

Remember that currently the "standard risk" patients have an approximate $25 \%$ death rate with the current regimen. That is in need of improvement. If you were to tell a parent: "Your child has only a $25 \%$ chance of dying with the less intensive treatment. The more intensive treatment being reserved for the higher risk patients, where it is proven to be better than the treatment that your child will get". I don't think anyone will agree to be randomized to the less intense treatment. However it makes little difference at this time with the more intense treatment being only the addition of etoposide and not higher dose ifosfamide. A $25 \%$ relapse rate is high risk also and all patients should be offered the most effective treatment available. After all, we don't offer less intense treatment to good risk osteosarcoma patients that have "low risk" small tibia primaries. We can take advantage of risk stratification to make radical changes in therapy for high risk patients, caution balanced by the expected almost $50 \%$ death rate in that high risk group.

The results for patients who present at diagnosis with bone metastases are uniformly dismal. Several attempts at high dose chemotherapy (HDC) with peripheral stem cell support have yielded 5 year EFS rates in the range $20 \%$ or less.

Prolonged ( 3 year) survival has been reported in these patients but eventually they relapse. The use of total body irradiation and the drugs busulfan and cyclophosphamide have caused severe, prolonged toxicity and even death due to drug toxicity. This regimen is a throw back to leukemia and lymphoma treatment and may not be optimal for solid tumors like Ewing tumor. Their toxicity also precludes repetition of the HDC treatment. Perhaps tandem auto-transplants while in complete remission (after primary tumor therapy) before the patient relapses, with an active regimen in relapsed Ewing sarcoma like high dose ifosfamide, etoposide, and carboplatinum (ICE) $[1,8,16]$ might be worth a feasibility study at some major European centers for those patients that present with bone metastases or inoperable primary tumors.

Whatever new curative plans are contemplated, certain facts remain. An EFS of even $75 \%$ for good risk patients is still an unacceptable tragedy for $25 \%$ of those patients. If we are to move forward in finding a greater percentage of cures we can try to do so with better use of existing therapies, which can be done immediately without the delay of the development of new drugs. Investigations into ways to ameliorate the toxic effects of drugs can also enhance the use of higher dose drug therapy. We still haven't come far enough to afford the luxury of "non-inferiority" studies to try to reduce the perceived threat of future toxicity. We can't take over 10 years time for an investigation that utilizes thousands of potential study subjects to try to prove non-inferiority of a regimen that is barely different than the current one. Thus, chemotherapy as we know it is still alive and well (and needed). Better ways to use old drugs can be as good as, if not better than, new unproven drugs. The reports of chemotherapy's exhaustion are premature.

It is hoped that continued successful investigations of chemotherapy, newer surgical reconstructive techniques, refinements in radiation oncology, as well as targeted and immunotherapies for Ewing tumor will continue to improve the outcome for these patients. 
The cooperative trial approach pioneered in Europe by Professor Heribert Jurgens will be the vehicle that builds on the established milestones in the treatment of this disease which were paved by his leadership.

\section{References}

1 Al-Faris N, Al Harbi T, Goia C et al. Does consolidation with autologous stem cell transplantation improve the outcome of children with metastatic or relapsed Ewing sarcoma? Pediatr Blood Cancer 2007; 49: $190-195$

2 Casey DA, Wexler LH, Merchant MS et al. Irinotecan and temozolomide for Ewing sarcoma: the Memorial Sloan-Kettering experience. Pediatr Blood Cancer 2009; 53: 1029-1034

3 Craft A, Cotterill S, Malcolm A et al. Ifosfamide-containing chemotherapy in Ewing's sarcoma: The Second United Kingdom Children's Cancer Study Group and the Medical Research Council Ewing's Tumor Study. J Clin Oncol 1998; 16: 3628-3633

4 Eilber FC, Eilber FR, Eckardt J et al. The impact of chemotherapy on the survival of patients with high-grade primary extremity liposarcoma. Ann Surg 2004; 240: 686-695 discussion 695-687

5 Granowetter $L$, Womer R, Devidas $M$ et al. Dose-intensified compared with standard chemotherapy for nonmetastatic Ewing sarcoma family of tumors: a Children's Oncology Group Study. J Clin Oncol 2009; 27: 2536-2541

6 Kushner BH, Kramer K, Modak S et al. Reduced risk of secondary leukemia with fewer cycles of dose-intensive induction chemotherapy in patients with neuroblastoma. Pediatr Blood Cancer 2009; 53: 17-22

7 Le Deley MC, Paulussen M, Lewis I et al. Cyclophosphamide Compared With Ifosfamide in Consolidation Treatment of Standard-Risk Ewing Sarcoma: Results of the Randomized Noninferiority Euro-EWING99R1 Trial. J Clin Oncol 2014, doi:10.1200/JCO.2013.54.4833

8 Milano GM, Cozza R, Ilari I et al. High histologic and overall response to dose intensification of ifosfamide, carboplatin, and etoposide with cyclophosphamide, doxorubicin, and vincristine in patients with high-risk Ewing sarcoma family tumors: the Bambino Gesu Children's Hospital experience. Cancer 2006; 106: 1838-1845
9 Nesbit ME Jr, Perez CA, Tefft M et al. Multimodal therapy for the management of primary, nonmetastatic Ewing's sarcoma of bone: an Intergroup Study. National Cancer Institute monograph 1981; 56: 255-262

10 Patel SR, Vadhan-Raj S, Papadopolous $N$ et al. High-dose ifosfamide in bone and soft tissue sarcomas: results of phase II and pilot studies-dose-response and schedule dependence. J Clin Oncol 1997; 15: 2378-2384

11 Paulussen M, Ahrens S, Dunst J et al. Localized Ewing tumor of bone: Final results of the cooperative Ewing's Sarcoma Study CESS 86. J Clin Oncol 2001; 19: 1818-1829

12 Paulussen M, Craft AW, Lewis I et al. Results of the EICESS-92 study: Two randomized trials of Ewing's sarcoma treatment - Cyclophosphamide compared with ifosfamide in standard-risk patients and assessment of benefit of etoposide added to standard treatment in high-risk patients. J Clin Oncol 2008; 26: 4385-4393

13 Rosen G, Caparros B, Nirenberg A et al. Ewings-Sarcoma - Ten-Year Experience with Adjuvant Chemotherapy. Cancer 1981; 47: 22042213

14 Skinner R, Cotterill SJ, Stevens MC. Risk factors for nephrotoxicity after ifosfamide treatment in children: a UKCCSG Late Effects Group study. United Kingdom Children's Cancer Study Group. Br J Cancer 2000; 82: $1636-1645$

15 Skinner R, Parry A, Price L et al. Glomerular toxicity persists 10 years after ifosfamide treatment in childhood and is not predictable by age or dose. Pediatr Blood Cancer 2010; 54: 983-989

16 Van Winkle P, Angiolillo A, Krailo $M$ et al. Ifosfamide, carboplatin, and etoposide (ICE) reinduction chemotherapy in a large cohort of children and adolescents with recurrent/refractory sarcoma: the Children's Cancer Group (CCG) experience. Pediatr Blood Cancer 2005; 44: $338-347$ 